\section{Treatment of stroke}

The fifth King's Fund Forum was held in London from 27 to 29 fune 1988. A panel of 12 listened to evidence from experts in public sessions attended by 250 people, including representatives of many professions, the public, and the press. After closed sessions the panel discussed its report with the audience and a consensus statement was then presented at a press conference.

\section{Consensus statement}

Every five minutes someone in the United Kingdom has a stroke. It is the cause of one in eight deaths and constitutes a formidable burden of disability and misery for patients, their carers, and the wider community. Half of all first strokes occur in people aged 75 and over, and given the continued rise in the number of very elderly people in the population stroke can be expected to remain an important source of suffering for the foreseeable future.

Despite these facts and their financial implicationsthe average health district in England and Wales spends at least $£ 3 \mathrm{~m}$ on stroke services each yearpolicy makers, members of the professions, and educators do not regard stroke as a high priority. There is no clear policy at district, regional, or national level on the appropriate planning, organisation, implementation, and evaluation of services for patients who have had a stroke and their carers. The services that are provided in hospital, primary care, and the community seem haphazard, fragmented, and poorly tailored to patients' needs, and there is a striking lack of convincing data on the effectiveness of widely used medical, psychological, and specific rehabilitative treatments. In the light of these deficiencies this consensus conference was set up to establish the appropriate responses to stroke in the acute phase and up to six months later. Some of our recommendations will continue to be relevant at later stages.

The panel comprised

Professor Anthony Clare (chairman), Professor Mike Drummond, Ms Annabel Ferriman, Mr Bryan Heiser, Ms Norah Lamb, Ms Jill Pitkeathley, Dr Ian Russell, Dr Simon Street, Professor Raymond Tallis, Dr John Todd, Ms Catherine van de Ven, and Professor Charles Warlow.

Invited experts presenting evidence were: $\mathrm{Mr}$ Robert Anderson, Ms Ann

Ashburn, Ms Janet Askham, Dr David Bainton, Dr John Bradshaw, Professor John Brocklehurst, Dr Rory Collins, Dr Christopher Davidson, Dr Pam Enderby, Dr Alan House, Ms Meryl

Hudson, Dr Richard

Langton Hewer, Dr Nadina

Lincoln, Ms Fiona Nouri,

Ms Pru Oswin, Professor

John Pathy, Dr Frank

Clifford Rose, Dr Peter

Sandercock, Mr Derek

Smith, Ms Joy Townsend,

and Dr Derick Wade. be developed. Such a service would develop a core team of nurses, therapists, social workers, and doctors with skills in meeting the needs of patients and their carers. It would also provide a necessary focus for the education of doctors, nurses, and other professionals and could be a resource for information.

Inpatients often suffer from being scattered throughout the hospital. In future they should be managed in one specific location. This approach would have the advantage of drawing together patients requiring similar treatment and rehabilitation and would facilitate the development of a mobile stroke team, which could span hospital and community. It could also provide day and respite care. Different models along these lines should be established, properly funded, and evaluated.

A high proportion of patients, however, are never admitted to hospital, while many of those who are admitted go in for social reasons. The district stroke policy should take into account the needs of those not admitted, together with the needs of their carers, many of whom are elderly themselves. It should also embrace patients in private and voluntary establishments.

There must be continuity of care between the hospital and community. This requires good liaison between health and local authorities at all levels. Patients should not be discharged until adequate preparation has been made for both the patient and carer. There should be realistic assessment of the carer's ability to look after a patient who has had a stroke before the patient is discharged. The carer should be given a genuine choice about arrangements. The patients and carers should participate fully at all times. Some patients are currently spending longer periods in hospital than their clinical state warrants owing to a shortage of appropriate facilities in the community. This is unacceptable.

Little information is given to patients and carers. All patients are entitled to a clear account of the nature and causes of their stroke; an honest discussion of the risks of recurrence, the speed and nature of recovery, and possible complications; and clear advice about the resumption of physical, social, and sexual activity. This should be assisted but not replaced by a clear fact sheet and other written material about stroke in general. Such material should be available in more than one language, and possibly in audiovisual form. More information rather than less should be given. Care should be taken not to hold back information unnecessarily, and the importance of listening to patients and carers is emphasised. Doctors can be overprotective towards patients, leading to resentment and complaints of unnecessary secrecy. Patients should also be put in touch with voluntary organisations such as stroke groups and the Chest, Heart and Stroke Association, and carers with the Carers' National Association.

When patients are not admitted to hospital the general practitioner has a key role in caring for the patients and in arranging any necessary diagnostic tests. After discharge the onus is also on the general practitioner to coordinate rehabilitation and continuing care services. If the general practitioner does not undertake this personally he or she should nominate a key worker to undertake these responsibilities. The key worker should be easily available to the patient and carers. It is the role of the general practitioner or key worker to fulfil the patient's information needs as outlined above, although all professionals have a responsibility to communicate fully at all times.

The general practitioner or key worker should 


\section{Routine}

\section{investigations}

Full blood count (including platelets)

Erythrocyte sedimentation rate

Urea and electrolyte concentrations

Glucose concentrations

Cholesterol concentrations

Syphilis tests

Sickling test (when appropriate)

Analysis of urine

Electrocardiography arrange to see and reassess the patient regularly. At the very least this will help to overcome the feelings of isolation and abandonment expressed by so many patients and carers. It would also enable the general practitioner to discover any further medical or social problems.

\section{Diagnosis of stroke}

An accurate history and a careful examination are paramount in the diagnosis of stroke. Tests should be directed to specific questions.

(1) Has the patient had a stroke?

(2) Is the stroke due to an infarct or haemorrhage?

(3) Is the stroke a manifestation of an underlying disease that requires treatment in its own right?

(4) Are there identifiable factors, such as high blood pressure, which if treated may reduce the chance of recurrence?

A neurological deficit of sudden onset in a patient who on clinical examination has signs compatible with a vascular lesion permits a confident diagnosis of stroke. In some cases, however, there may be an inadequate history (as in a patient who lives alone or who has impairment of consciousness). The differential diagnosis will then include other intracranial disease and the various causes of coma.

Stroke may be a manifestation of an underlying disorder-for example, common diseases such as diabetes or rarer conditions such as cranial arteritis and infective endocarditis. The clinical features will direct suspicions, but screening tests should be undertaken routinely in all cases (see box). Chest radiography may also be required. These routine tests will also cover identifiable risk factors, whose correction may be of benefit in preventing recurrence.

The differentiation of haemorrhage from infarct is under certain circumstances critical. It is impossible to do this clinically and the best way of determining the type of stroke is computed tomography within two weeks. The box overleaf shows the main indications for computed tomography. Scanning can be undertaken as an outpatient investigation. There is a strong case for scanning most patients who present with a presumptive stroke, excluding those in whom antiplatelet treatment is contraindicated. The resources required to scan this large group of patients may well be outweighed by a reduction in the recurrence of strokes and myocardial infarcts and in the associated costs of health care. Currently only half of all health districts have a computed tomographic scanner. None the less, local access to scanning facilities should be an essential part of a district stroke service.

Lumbar puncture should be used only when meningitis is suspected or subarachnoid haemorrhage cannot be diagnosed by computed tomography. Isotope brain scanning is appropriate only when subdural haematoma has to be excluded and computed tomography is not available. Magnetic resonance imaging is a sensitive method of imaging the brain, but it has not yet been proved to be of great practical value in strokes. The use of angiography in an ischaemic stroke is usually linked to the intention to carry out a carotid endarterectomy, but ultrasound scanning as a preliminary screen will reduce this requirement. Echocardiography should be carried out when there is a strong clinical suspicion of cardiac embolic stroke and anticoagulation or other specific treatment is seriously considered

\section{Effective treatments in the first few days after stroke}

No treatment has conclusively been shown to be effective in limiting the neuronal damage associated with stroke. Small randomised trials have suggested that some drugs (notably glycerol, naftidrofuryl, and nimodipine) may be effective. There is insufficient evidence to justify the use of these drugs outside large randomised trials.

Neurosurgery may be indicated in a few patientsfor example, those with a cerebellar haematoma or a haemorrhage due to aneurysm or arteriovenous malformation. A few strokes will be due to an underlying disease that should respond to treatment-for example, cranial arteritis, myelomatosis, polycythaemia, thrombocytopenia, or infective endocarditis. Care should be taken to avoid early complications of stroke, including chest infections and other causes of hypoxia, venous thromboembolism, urinary infections and incontinence, dehydration, constipation, pressure sores, falls and injuries, painful shoulder, and spasticity.

\section{Treatment to reduce recurrence}

There is strong scientific evidence that antiplatelet treatment for ischaemic strokes reduces recurrence. We recommend low doses of aspirin (150-300 mg daily). In the absence of computed tomography to exclude intracerebral haemorrhage, prophylactic aspirin may still have a favourable ratio of benefit to risk but cannot be safely recommended.

There is also evidence that reducing high blood pressure and high blood cholesterol concentrations reduces the risks of subsequent vascular events. Nevertheless, it is important that hypotensive treatment should not be too vigorous or begin too early because of the risk of reducing the blood flow to the brain.

Anticoagulation treatment is often used for ischaemic stroke associated with atrial fibrillation, cardiac valve disease, myocardial infarction with presumed mural thrombus, or carotid stenosis. Although this has theoretical appeal, there is little supportive scientific evidence. Similarly, carotid endarterectomy is often performed for carotid artery disease, with even less empirical support. Randomised trials addressing some of these issues are currently in progress.

Excessive consumption of alcohol should be reduced to facilitate the control of high blood pressure. Patients should be encouraged to stop smoking as this may reduce cerebrovascular events and will certainly reduce ischaemic cardiac events.

\section{Assessment and rehabilitation during the first six months}

Multidisciplinary assessment should begin at once and rehabilitation as early as possible. In addition to a precise definition of neurological deficit assessment should cover motor and sensory function, swallowing, cognitive function and mood, communication skills, and performance of activities of daily living. For carer as well as patient assessment should include general health, psychological and social problems, the support network, housing, finance, employment, and leisure activities.

Assessments according to agreed standards must be done at regular intervals, the exact interval being determined for each person. Certain principles should be applied throughout the rehabilitation of patients. These include documenting impairments, disabilities, and handicaps and when possible measuring them with simple valid scales; maximising independence and minimising learned dependency; and taking account of the whole person and the environment.

Participants in the rehabilitation process include nurses, physiotherapists, occupational therapists, speech therapists, dietitians, chiropodists, social workers, psychologists, and doctors. They should 


Main indications
for computed
tomography
Uncertain diagnosis
of stroke
Current or
contemplated
anticoagulation or
antiplatelet
treatment, or both
- Suspected cerebellar
haematoma
- Possible carotid
endarterectomy
Suspected
subarachnoid
haemorrhage
Young patient

work as a team. For the team to work effectively there must be trust and respect for each other's skill. A key worker should be identified for every patient at every stage to coordinate an individual plan and provide education and positive support. The professional's role is not only to assess and treat but to provide education and positive support to carers.

Throughout the period of rehabilitation the key worker has a responsibility to keep patients informed of the nature of their problems and carers should actively participate in the entire process. The prospects for improvement and the goals of treatment must be discussed with patients and, when appropriate, carers.

The main problems during rehabilitation need to be addressed. These include $(a)$ misunderstandings and rivalries among professionals; $(b)$ breakdown of communication among professionals, patients, and carers; (c) insufficient appreciation of the impact of stroke on the patient's family; $(d)$ ill prepared and sometimes unplanned discharge home; $(e)$ serious shortage of treatment; $(f)$ long periods in which patients are unoccupied; $(g)$ ill considered admission to hospital; (h) failure to recognise and respond to mood disturbances; $(i)$ delegation of care to inadequately trained medical staff; and $(j)$ confusion caused by the participation of too many people.

There is no doubt that many consumers derive considerable satisfaction from the general stimulating effect of treatment and the practical benefit of much of the advice given. It is clear that the rehabilitation process can be effective, but there is little evidence of which aspects are beneficial. The personal and professional skills of therapists must not be undervalued. Many therapists are themselves keen to evaluate their work rigorously and to identify those components which are most effective. We support them.

\section{Principles of good practice in providing services}

Stroke care should be reorganised to take account of the following points:

Standards must be agreed in collaboration with all the professionals concerned and take account of the views of patients and carers. The service must be strongly led and coordinated, cost effective, and kept under regular review, bearing in mind the many different professional groups participating. The service should be planned at district level to include primary and community as well as hospital services. When there is a joint care planning team this should be one of its prime responsibilities.

The delivery of care must be monitored to ensure that the agreed standards are being implemented for individual patients. Monitoring should include consumers' views.

For most patients the stay in hospital is a short prelude to a lifelong disability. In considering the balance between hospital and community care it should be remembered that costs and benefits fall not only on the NHS and other agencies but above all on patients and their families. An effective community service must take account of this and plan accordingly. As the needs of individual patients and carers vary there should be flexibility in the provision of community care, both after the onset of stroke and after discharge from hospital. In particular, attention should be paid to integrating hospital and community care at all times.

New organisational structures and changes in budgetary systems for stroke services may be required to encourage this flexibility. One approach could involve the named person responsible for implementing and monitoring a district's stroke policy. This person could have budgetary responsibility and be able to buy individual components of care, including those provided by the voluntary sector.

There has been little evaluation of the effectiveness and cost of most components of care. This is urgently needed. In evaluating treatments, or even packages of care, the randomised trial is almost always the most effective method provided that appropriate outcomes are measured, including the satisfaction of patients. Sometimes, however, other research methods might be more practical and appropriate. Multiprofessional approaches to research should also be encouraged. Research should be seen as part of the career development of all professionals and not just of hospital doctors. In particular, therapists need to develop research skills and this will require access to research funds and, more importantly, training in research methodology. The lack of evaluation can be rectified only be earmarking research funds in proportion to the health care resources devoted to stroke.

Education and training should be provided for all professionals, carers, and patients to increase their knowledge about stroke, improve their skills in dealing with the condition, and change attitudes so that the needs of patients with stroke are given higher priority and more status. Additional resources must be provided. It is important to raise the awareness of politicians, the public, and the media, and a programme of education and promotion of what needs to be done should be undertaken.

All the recommendations in this consensus statement could and should be accomplished within the present system of health care in the United Kingdom. The quickest and most effective way to implement these recommendations would be the formation of integrated district stroke services to encompass care, education, and research at every level both in hospitals and in the community. To ensure that such a service can be implemented health authorities must provide sufficient manpower and resources.

The cost of better services for patients with stroke will increase, but this could be considerably offset by concentrating existing resources, avoiding unnecessary investigations and ineffective treatments, and setting and monitoring standards of care.

\section{Update box for Oxford Handbook of Clinical Medicine, p 185}

\section{Preventing hepatitis $B$ and cirrhosis, chronic hepatitis, and hepatic neoplasia associated with hepatitis $B$}

Use hepatitis B vaccine (H-B-Vax or Engerix B; Engerix $B$ is genetically engineered and cheaper but not yet recommended for those under 3 years old).

Dose: $1 \mathrm{ml}$ into deltoid (or the anterolateral thigh in children), repeated at 1 and 6 months (child: $0.5 \mathrm{ml} \times 3$ of H-B-Vax; neonates: see Oxford Handbook of Clinical Specialties, p 208).

Indications: Populations at risk: health workersfor example, nurses, hospital doctors and general practitioners, and dentists-haemophiliacs, homosexuals, abusers of intravenous drugs, patients receiving haemodialysis, and the sexual partners of known carriers of hepatitis $\mathrm{B}$ e antigen. Patients who are immunocompromised may need further doses.

Contraindications: Any severe febrile infection.

Antibody protection may be checked one month after the last dose. Values eventually begin to wane so consider revaccination after three to five years.

It is important to note that protective immunity begins about six weeks after the first immunising dose so hepatitis B vaccine is inappropriate for those who have had recent exposure. For such people specific antihepatitis B immunoglobulin is the preferred treatment if they have not previously been immunised. -J M LONGMORE

Principal source: British National Formulary 1988;14.4. 